\title{
Quantum control with spectral constraints
}

\author{
Lukasz Pawela • Zbigniew Puchała
}

Received: 1 May 2012 / Accepted: 18 September 2013 / Published online: 6 October 2013

(C) The Author(s) 2013. This article is published with open access at Springerlink.com

\begin{abstract}
Various constraints concerning control fields can be imposed in the realistic implementations of quantum control systems. One of the most important is the restriction on the frequency spectrum of acceptable control parameters. It is important to consider the limitations of experimental equipment when trying to find appropriate control parameters. Therefore, in this paper, we present a general method of obtaining a piecewise-constant controls, which are robust with respect to spectral constraints. We consider here a Heisenberg spin chain; however, the method can be applied to a system with more general interactions. To model experimental restrictions, we apply an ideal low-pass filter to numerically obtained control pulses. The usage of the proposed method has negligible impact on the control quality as opposed to the standard approach, which does not take into account spectral limitations.
\end{abstract}

Keywords Quantum information - Quantum computation · Control in mathematical physics

\section{Introduction}

One of the fundamental issues of the quantum information science is the ability to manipulate the dynamics of a given complex quantum system. Since the beginning of quantum mechanics, controlling a quantum system has been an implicit

\footnotetext{
Ł. Pawela $(\bowtie) \cdot$ Z. Puchała

Institute of Theoretical and Applied Informatics, Polish Academy of Sciences,

Bałtycka 5, 44-100 Gliwice, Poland

e-mail: lukasz.pawela@gmail.com

Z. Puchała

e-mail: z.puchala@iitis.pl
} 
goal of quantum physics, chemistry, and implementations of quantum information processing.

If a given quantum system is controllable, i.e., it is possible to drive it into a previously fixed state, it is desirable to develop a control strategy to accomplish the required control task. In the case of finite dimensional quantum systems, the criteria for controllability can be expressed in terms of Lie-algebraic concepts [1-3]. These concepts provide a mathematical tool, in the case of closed quantum systems, i.e., systems without external influences.

It is an important question whether the system is controllable when the control is performed only on a subsystem. This kind of approach is called a local-controllability and can be considered only in the case when the subsystems of a given system interact. As examples may serve coupled spin chains or spin networks [2,4-6]. Local-control has a practical importance in proposed quantum computer architectures, as its implementation is simpler and the effect of decoherence is reduced by decreased number of control actuators $[7,8]$.

A widely used method for manipulating a quantum system is a coherent control strategy, where the manipulation of the quantum states is achieved by applying semiclassical potentials in a fashion that preserves quantum coherence. In the case when a system is controllable, it is a point of interest what actions must be performed to control a system most efficiently, bearing in mind limitations imposed by practical restrictions. Various constraints concerning control fields can be imposed in the realistic implementations of quantum control systems. One of the most important is the restriction on the frequency spectrum of acceptable control parameters. Such restrictions come into play, for example, in an experimental setup that utilizes an external magnetic field [9]. In the case of such systems, due to various limitations, the application of piecewise-constant controls is not accurate. The real realization of controls is somehow smoothed by some filter induced by an experimental limitations. Thus, it is reasonable to seek control parameters in the domain imposed by the experimental restrictions.

In article [10], there has been discussed how the low-pass filtering, i.e., eliminating high-frequency components in a Fourier spectra, on a numerically obtained optimal control pulses affects a quality of performed control. This approach makes a contact with experimental realizations, since it implements the limitations or real quantum control systems.

In this paper, we present a general method of obtaining a piecewise-constant controls, which is robust with respect to low-pass filtering. The above means that elimination of high frequencies in a Fourier spectra reduces the fidelity only by a small amount. We utilize this approach to obtain numerically control pulses on a Heisenberg spin chain [10-12]; however, it can be applied to a quantum system with more general interactions.

This paper is organized as follows. In Sect. 2, we provide a general description of a quantum mechanical control system. In Sect. 3, we provide the description of the simulation setup used to test our model. Section 4 contains results obtained from numerical simulations and their discussion. In Sect. 5, we provide a summary of the presented work and give some concluding remarks. 


\section{Our model}

To demonstrate a method of obtaining piecewise-constant controls, which are robust with respect to low-pass filtering, we will consider an isotropic Heisenberg spin$1 / 2$ chain of a finite length $N$. The control will be performed on the first spin only. The total Hamiltonian of the aforementioned quantum control system is given by

$$
H(t)=H_{0}+H_{c}(t)
$$

where

$$
H_{0}=J \sum_{i=1}^{N-1} S_{x}^{i} S_{x}^{i+1}+S_{y}^{i} S_{y}^{i+1}+S_{z}^{i} S_{z}^{i+1},
$$

is a drift part given by the Heisenberg Hamiltonian. The control is performed only on a first spin and is Zeeman-like, i.e.,

$$
H_{c}(t)=h_{x}(t) S_{x}^{1}+h_{y}(t) S_{y}^{1}
$$

In the above, $S_{k}^{i}$ denotes $k^{\text {th }}$ Pauli matrix acting on the spin $i$. Time-dependent control parameters $h_{x}(t)$ and $h_{y}(t)$ are chosen to be piecewise constant. We will refer to the values of the control pulses in the $i$ th time interval as $h_{x, i}$ and $h_{y, i}$. When we reference a general control pulse without specifying the direction, we will write $h_{l}$ and the corresponding control Hamiltonian will be denoted $H_{l}$. Furthermore, as opposed to [10], we do not restrict the control fields to be alternating with $x$ and $y$, i.e., they can be applied simultaneously (see e.g. [13] for similar approach). For notational convenience, we set $\hbar=1$, and after this, rescaling frequencies and control field amplitudes can be expressed in units of the coupling strength $J$, and on the other hand, all times can be expressed in units of $1 / J$ [10].

The system described above is operator controllable, as it was shown in [5] and follows from a controllability condition using a graph infection property introduced in the same article. The controllability of the described system can be also deduced from a more general condition utilizing the notion of hypergraphs [6].

Since the interest here is focused on operator control, a quality of a control will be measured with the use of gate fidelity,

$$
F=\frac{1}{2^{N}}\left|\operatorname{Tr}\left(U_{T}^{\dagger} U(h)\right)\right|
$$

where $U_{T}$ is the target quantum operation and $U(h)$ is an operation achieved by control parameters $h$. We choose gate fidelity as it neglects global phases.

To obtain piecewise-constant controls, which are robust with respect to low-pass filtering we will minimize the power in the high frequency part of a controls Fourier spectrum. We will do so by minimizing the following functional 


$$
G=(1-\mu) P-\mu F,
$$

where $F$ is the gate fidelity described above, $\mu$ is a weight assigned to fidelity and $P$ is a contribution of high frequencies in the total power of the control parameters. The above can be stated as, if $y$ is a vector of Fourier coefficients for a control parameters $h$ of length $n$, i.e.

$$
y=Q h, \text { where } Q=n^{-1 / 2}\left\{\mathrm{e}^{2 \pi \mathrm{i} k l / n}\right\}_{k, l=0}^{n-1},
$$

then $P$ is given by

$$
P=\frac{\sum_{i=\frac{n}{2}-\Delta}^{\frac{n}{2}+\Delta}\left|y_{i}\right|^{2}}{|y|^{2}},
$$

for some $\Delta$ related to the cutoff frequency of low-pass filter.

\section{Simulation setup}

To demonstrate the beneficialness of our approach, we study three- and four-qubit spin chains. The control field is applied to the first qubit only. Our target gates are:

$$
\operatorname{NOT}_{N}=\mathbb{1}^{\otimes N-1} \otimes \sigma_{x},
$$

the negation of the last qubit of the chain, and

$$
\operatorname{SWAP}_{N}=\mathbb{1}^{\otimes N-2} \otimes \mathrm{SWAP}
$$

swapping the states between the last two qubits.

For each of these cases, we find two sets of control parameters. One with frequency constraints and one without. Next, we calculate an appropriate filter and using these filtered values of control parameters, we calculate the fidelity of the quantum operation. In each case, 120 independent sets of control parameters were found.

We provide an explicit example in which we set the duration of the control pulse to $\Delta t=0.2$ and the total number of pulses in each direction to $n=128$ for the three-qubit chain and $n=512$ in the four-qubit case, although the presented method may be applied for arbitrary values of $\Delta t$ and $n$. The weight of fidelity in Eq. (5) is set to $\mu=1$ in the unconstrained case and to $\mu=0.05$ in the constrained case. Although the weight of the fidelity is small, the optimization still yields high fidelity values while maintaining low contribution of high frequencies in the power spectrum. We set the cutoff frequency in Eq. (7) to $\Delta=\frac{n}{4}$.

The applied filter is a frequency filter with the cutoff frequency equal to the frequency discriminated by the functional (5). As an example, we consider this filter 
to be an ideal low-pass filter, which was previously studied, e.g., in [11]. Obviously, one can use other filters. A more general discussion of spectral filtering is presented in [14]. The ideal low-pass filter leaves the frequencies only in the interval $\left[-\omega_{0}, \omega_{0}\right]: f(\omega)=\Theta\left(\omega+\omega_{0}\right)-\Theta\left(\omega-\omega_{0}\right)$, where $\Theta$ is the Heaviside step function. We obtain the following expressions for the filtered control parameters [11]:

$$
\hat{h}_{k}(t)=\frac{1}{\pi} \sum_{i=1}^{n} h_{k, i}\left[a_{i+1}(t)-a_{i}(t)\right]
$$

where $k \in\{x, y\}$ and $t$ denotes time. In other words, $\hat{h}_{k}(t)$ is a filtered version of the control fields $h_{k}$ at the time moment $t$.

$$
\begin{aligned}
& a_{n}(t)=\operatorname{Si}\left[\omega_{0}(n \Delta t-t)\right], \\
& \operatorname{Si}(x)=\int_{0}^{x}(\sin t / t) \mathrm{d} t .
\end{aligned}
$$

The calculation of the gradient of the fidelity function can be found in the work by Machnes et al. [15]. Here, we only show the final result of the calculation

$$
\frac{\partial F\left(U_{k}\right)}{\partial h_{l}}=\frac{1}{N} \Re \operatorname{Tr}\left\{\mathrm{e}^{-\mathrm{i} \phi} U_{T}^{\dagger} U_{n} \ldots U_{k+1} \frac{\partial U_{K}}{\partial h_{l}} U_{k-1} U_{1}\right\},
$$

where

$$
\mathrm{e}^{-\mathrm{i} \phi}=\frac{\overline{\operatorname{Tr}\left(U_{T}^{\dagger} U(h)\right)}}{F}
$$

and $\frac{\partial U_{K}}{\partial h_{l}}$ may be computed using the following formula [15, Equation (24)]

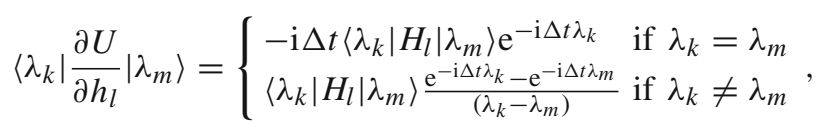

where $U$ is the unitary gate implemented by the control pulses

$$
U=\exp (-\mathrm{i} \Delta t H)=\exp \left(-\mathrm{i} \Delta t\left(H_{0}+\sum_{l} h_{l} H_{l}\right)\right) .
$$

In this case, $H_{l}$ is the control Hamiltonian corresponding to the control pulse $h_{l} \cdot|\lambda\rangle$ and $\lambda$ are the eigenvectors and eigenvalues of the total Hamiltonian of the system.

We conduct our simulations using the Broyden-Fletcher-Goldfarb-Shanno (BFGS) method [16]. This method is commonly used in quantum control theory for optimization of control pulses $[10,11,17]$. We first choose an initial guess for the 
control field vectors. The algorithm then generates iteratively new control field vectors such that at each iteration point, the fidelity is increased. The algorithm terminates after a desired accuracy is reached. This procedure ensures convergence to a local maximum, but does not guarantee the convergence to a globally optimal sequence. Hence, we perform a number of simulations, using different initial conditions.

To calculate the gradient of $P$, one should note an elementary fact concerning differentiation of vector valued functions. For a real vector $h$, we define $y=A h$ for some fixed matrix $A$. Straightforward calculations give us

$$
\frac{\partial\left|y_{k}\right|^{2}}{\partial h_{l}}=2 \Re\left(\bar{A}_{k l} y_{k}\right) .
$$

In the case when matrix is a quantum Fourier transform gate $A=Q$ defined in Eq. (6), we obtain that

$$
\frac{\partial\left|y_{k}\right|^{2}}{\partial h_{l}}=2 \Re\left(\bar{Q}_{k l} y_{k}\right)=\frac{2}{\sqrt{n}} \Re\left(\mathrm{e}^{-2 \pi \mathrm{i} k l / n} y_{k}\right) .
$$

This calculation is used to find the gradient of the contribution of high frequencies in the total power given by Eq. (7).

\section{Results}

Figure 1a shows a plot of the control parameters for the target gate $U_{T}=\mathrm{NOT}_{3}$ before and after applying the frequency filter. These parameters were found using with the value of the weight $\mu=1$, resulting in no penalty for high-frequency terms. Clearly, the signal after filtering differs from the original values. This is reflected in the values of the fidelity of the operation. Before filtering, the fidelity is $F>1-10^{-12}$; however, after filtering, the value drops to $F=0.85$.

Next, in Fig. 1b, we show the plot of the control parameters for the target gate $U_{T}=\mathrm{NOT}_{3}$, before and after applying the frequency filter. Only this time, the controls were found using the value of the weight $\mu=0.05$ resulting in a penalty for highfrequency terms. A short glance reveals that the filtered parameters are almost the same as the original ones. This is reflected by the fidelity of the operation. Before filtering, the fidelity is $F>1-10^{-9}$, and after filtering, it drops only to $F>1-10^{-2}$, which is still a satisfactory value. Hence, these sets of control parameters are well-suited for use in computations.

Figure 2a, b shows analogical results for the target gate $U_{T}=\mathrm{SWAP}_{3}$. The qualitative results in this case are the same as for the $\mathrm{NOT}_{3}$ target gate discussed earlier. Fidelity values before and after filtering are the same order as for the $\mathrm{NOT}_{3}$ gate. Again, we reach a conclusion that these sets of control parameters are well suited for use in computations. 

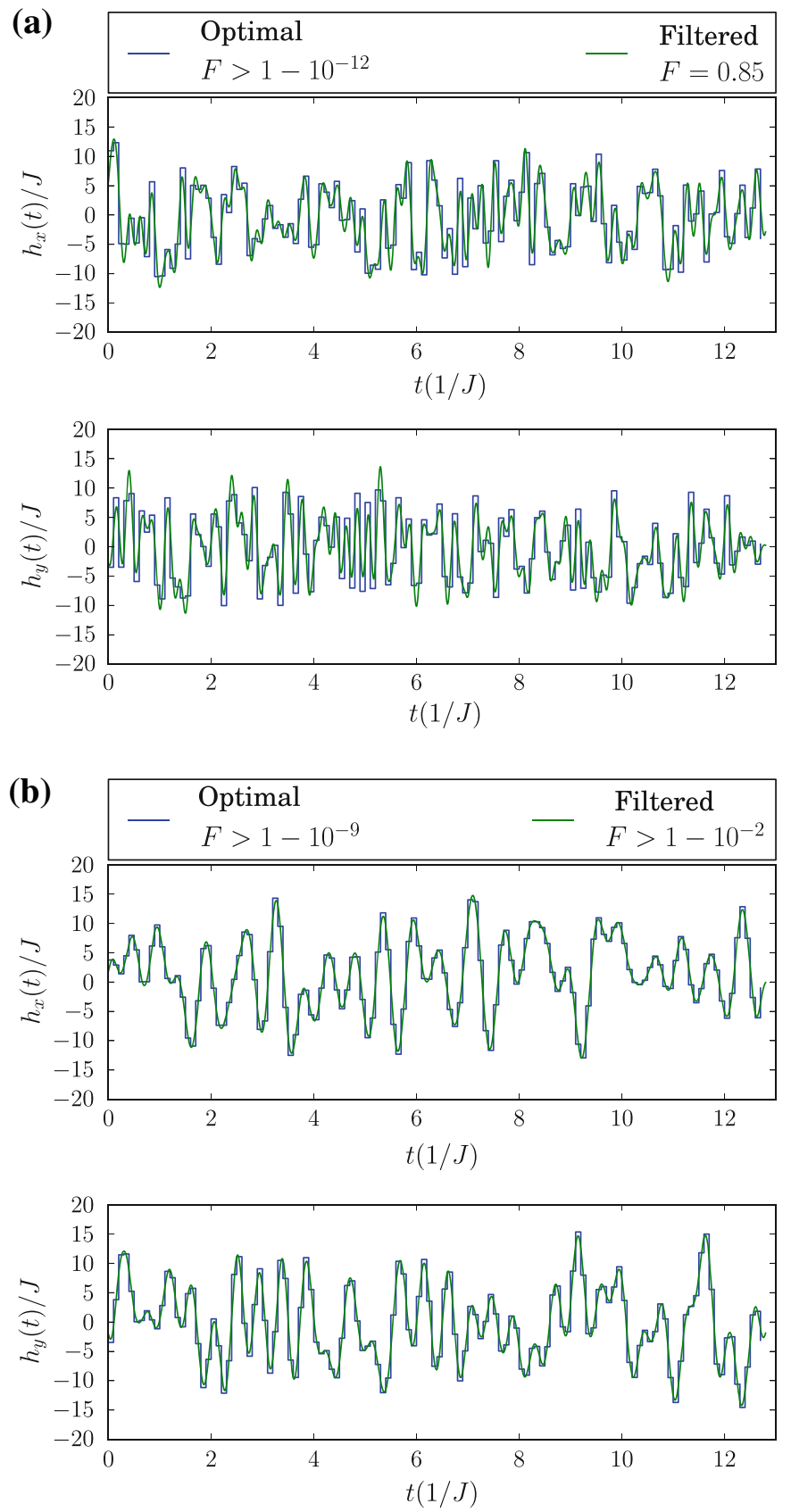

Fig. $1 x$ and $y$ components of the control field for target gate $U_{T}=\mathrm{NOT}_{3}$. a Unconstrained case. b Constrained case 

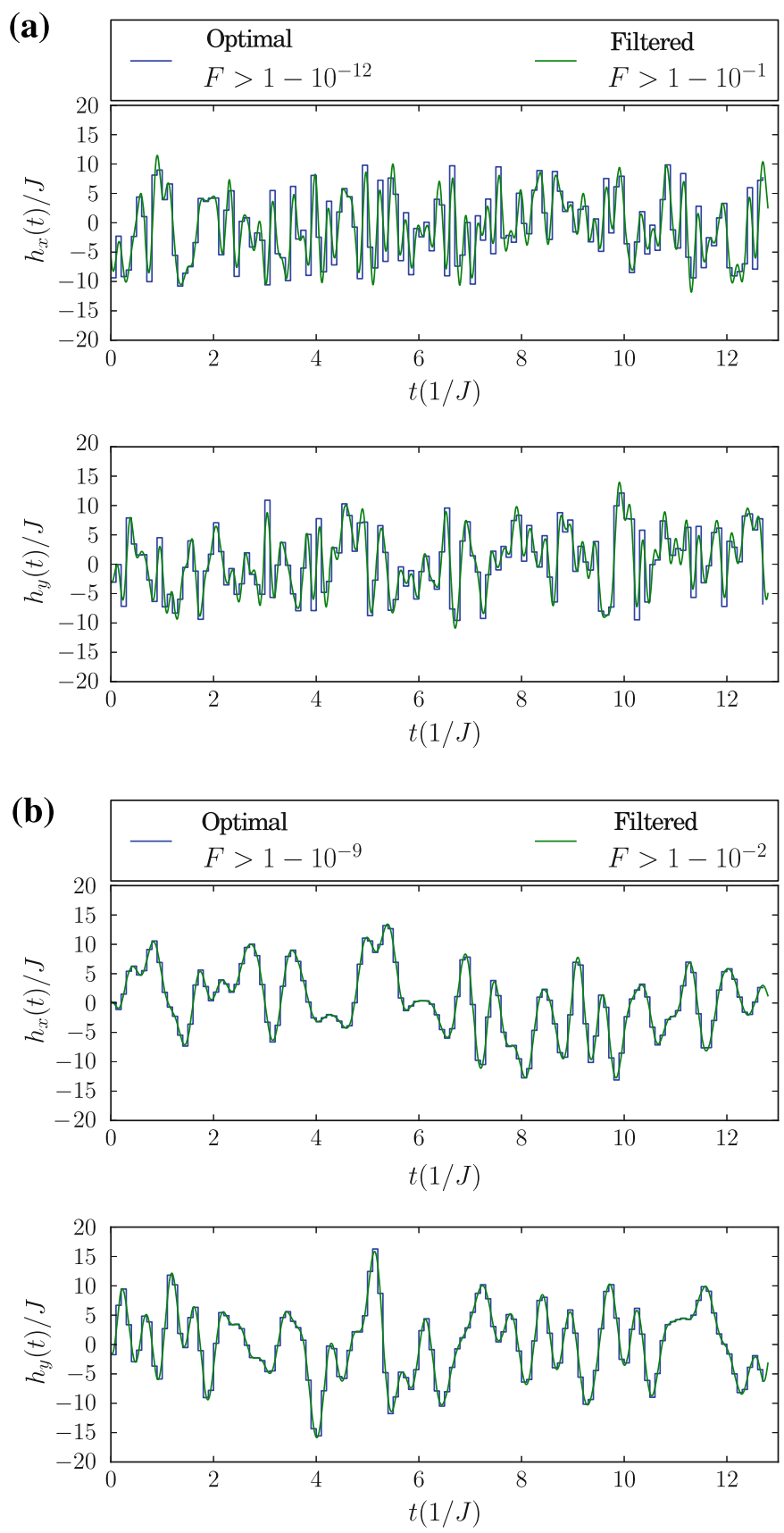

Fig. $2 x$ and $y$ components of the control field for target gate $U_{T}=\mathrm{SWAP}_{3}$. a Unconstrained case. b Constrained case 

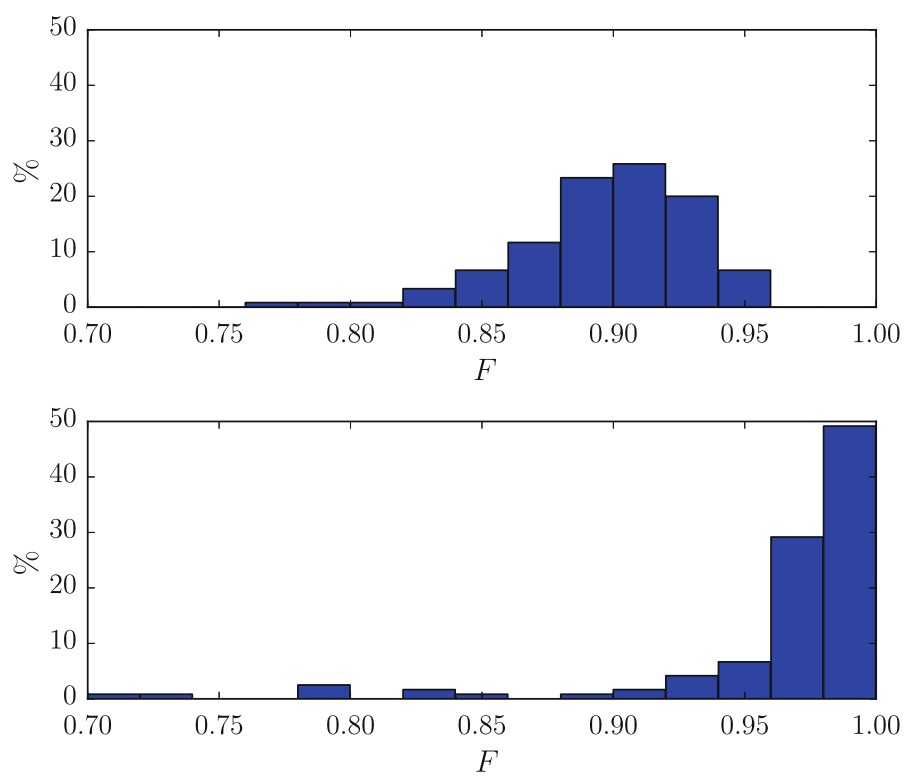

Fig. 3 A histogram of the fidelities after filtering. Top without considering spectral constraints, bottom with spectral constraints considered. Target gate $U_{T}=\mathrm{NOT}_{3}$

Results obtained for the four-qubit chain (not shown here) qualitatively resemble the results for the three-qubit chain. In this case, a penalty for high-frequency terms of the control parameters also leads to higher fidelities after filtering.

Figure 3 depicts the histograms of fidelities of filtered control parameters for the $\mathrm{NOT}_{3}$ gate for different initial vectors of control fields. These vectors are drawn at random; coordinates are stochastically independent and have a uniform distribution on an interval $[-10,10]$. The top plot shows the histograms for control parameters found without spectral constraints and the bottom one shows what happens when one takes spectral constraints into consideration. Clearly, a typical set of control parameters has a higher fidelity of operation when one considers spectral constraints in the optimization phase. Strictly speaking, around $80 \%$ of the control parameter sets have a fidelity greater than 0.96 , whereas in the unconstrained case, there are no control parameter sets that have such high fidelities. These facts lead to a conclusion that optimization with spectral constraints will lead to high fidelity of experimental realizations of the $\mathrm{NOT}_{3}$ gate.

Analogical results for the $\mathrm{SWAP}_{3}$ are shown in Fig. 4. The top plot shows the histograms for control parameters found without spectral constraints, and the bottom one shows what happens when one takes spectral constraints into consideration. In this case, around $75 \%$ of all constrained control parameter sets have a fidelity higher than 0.96 after filtering. Also, there are no unconstrained control parameter sets with fidelities in this range. Hence, spectral constraints imposed during the optimization step have led to control parameters far less sensitive to experimental equipment limitations. 

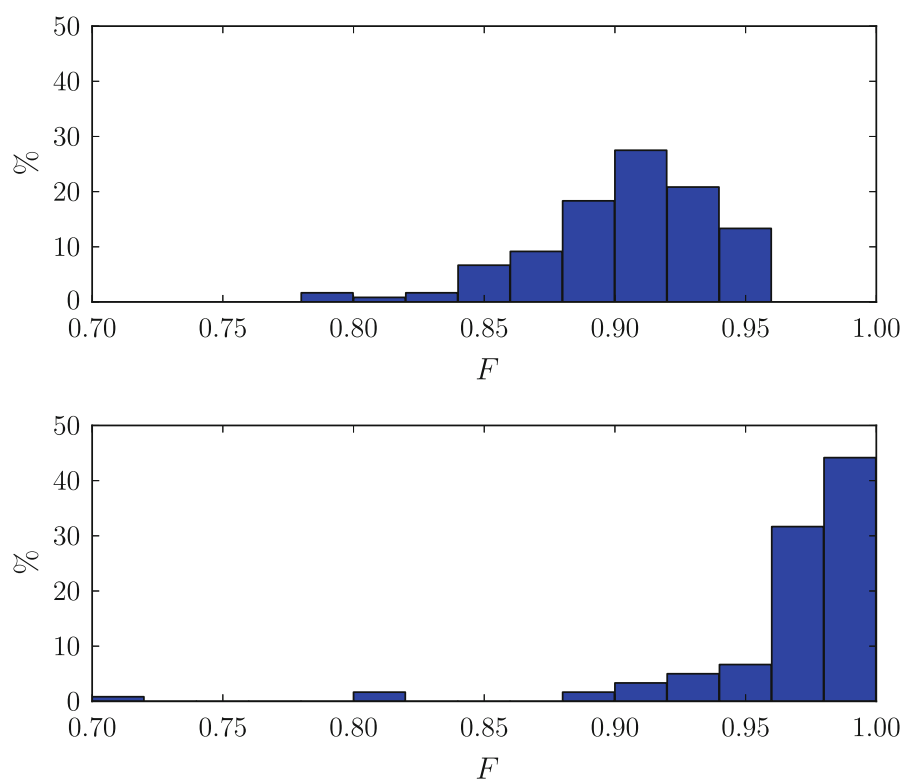

Fig. 4 A histogram of the fidelities after filtering. Top without considering spectral constraints, bottom with spectral constraints considered. Target gate $U_{T}=\mathrm{SWAP}_{3}$

\section{Conclusions}

We investigated the impact of spectral constraints imposed on the control parameters of a quantum operation on the fidelity of the quantum operation which they implement. In order to compare our approach with the unconstrained case, we apply an ideal low-pass filter to the control parameters.

We have shown that imposing spectral constraints on the control parameters leads to higher average fidelity of the quantum operation after appropriate filtering, than in the unconstrained case. These results are independent of the type of quantum operation and the number of qubits in the system under consideration.

Furthermore, the requirement for smooth control parameters does not result in the increase in time necessary to conduct a quantum operation. Comparing with other research in the field [11], our times are on the same order.

Further work on this subject might take into account more subtle parameters of the experimental setup than the frequency cutoff of signal sources. For instance, one could wish to find control parameters that are far from transient characteristics of the experimental setup. This may lead to an enhancement of fidelities of operations achieved experimentally by eliminating unwanted signal roughness.

Acknowledgments Work by $Ł$. Pawela was partially supported by the Polish National Science Centre under the Grant Number N N514 513340. Z. Puchała was partially supported by the Polish Ministry of Science and Higher Education under the project number IP2011 044271. The development of numerical methods was supported by the Grant N N514 513340. Analytical studies were supported by the project IP2011 044271. Numerical simulations presented in this work were performed on the "Leming" and "Swistak" computing systems of The Institute of Theoretical and Applied Informatics, Polish Academy of Sciences. 
Open Access This article is distributed under the terms of the Creative Commons Attribution License which permits any use, distribution, and reproduction in any medium, provided the original author(s) and the source are credited.

\section{References}

1. Albertini, F., D’Alessandro, D.: The Lie algebra structure and controllability of spin systems. Linear Algebra Its Appl. 350, 213 (2002). ISSN 0024-3795, http://www.sciencedirect.com/science/article/ pii/S0024379502002902

2. d'Alessandro, D.: Introduction to Quantum Control and Dynamics. Chapman \& Hall, London (2008)

3. Elliott, D.: Bilinear Control Systems: Matrices in Action. Springer, Berlin (2009). ISBN 1402096127

4. Burgarth, D., Giovannetti, V.: Full control by locally induced relaxation. Phys. Rev. Lett. 99, 100501 (2007). doi:10.1103/PhysRevLett.99.100501

5. Burgarth, D., Bose, S., Bruder, C., Giovannetti, V.: Local controllability of quantum networks. Phys. Rev. A 79, 60305 (2009). ISSN 1094-1622, doi:10.1103/PhysRevA.79.060305

6. Puchała, Z.: Local controllability of quantum systems. Quantum Inf. Process. (2012). doi:10.1007/ s11128-012-0391-X

7. Montangero, S., Calarco, T., Fazio, R.: Robust optimal quantum gates for Josephson charge qubits. Phys. Rev. Lett. 99, 170501 (2007). doi:10.1103/PhysRevLett.99.170501

8. Fisher, R., Helmer, F., Glaser, S.J., Marquardt, F., Schulte-Herbrüggen, T.: Optimal control of circuit quantum electrodynamics in one and two dimensions. Phys. Rev. B 81, 085328 (2010). doi:10.1103/ PhysRevB.81.085328

9. Chaudhury, S., Merkel, S., Herr, T., Silberfarb, A., Deutsch, I., Jessen, P.: Quantum control of the hyperfine spin of a Cs atom ensemble. Phys. Rev. Lett. 99, 163002 (2007). doi:10.1103/PhysRevLett. 99.163002

10. Heule, R., Bruder, C., Burgarth, D., Stojanović, V. M.: Local quantum control of Heisenberg spin chains. Phys. Rev. A 82, 052333 (2010). doi:10.1103/PhysRevA.82.052333

11. Heule, R., Bruder, C., Burgarth, D., Stojanović, V.M.: Controlling qubit arrays with anisotropic XXZ Heisenberg interaction by acting on a single qubit. Eur. Phys. J. D 63, 41 (2011), ISSN 1434-6060, 1434-6079, http://www.springerlink.com/content/b4rr1wg3400442lv/

12. Miszczak, J., Gawron, P., Puchała, Z.: Qubit flip game on a Heisenberg spin chain. Quantum Inf. Process. (2012). doi:10.1007/s11128-011-0322-2

13. Khaneja, N., Reiss, T., Kehlet, C., Schulte-Herbrüggen, T., Glaser, S.J.: Optimal control of coupled spin dynamics: design of NMR pulse sequences by gradient ascent algorithms. J. Magn. Reson. 172, 296 (2005), ISSN 1090-7807, http://www.sciencedirect.com/science/article/pii/S1090780704003696

14. Werschnik, J., Gross, E.: Quantum optimal control theory. J. Phys. B: Atom. Molecul. Opt. Phys. 40, R175 (2007)

15. Machnes, S., Sander, U., Glaser, S.J., de Fouquières, P., Gruslys, A., Schirmer, S., Schulte-Herbrüggen, T.: Comparing, optimizing, and benchmarking quantum-control algorithms in a unifying programming framework. Phys. Rev. A 84, 022305 (2011). doi:10.1103/PhysRevA.84.022305

16. Press, W., Flannery, B., Teukolsky, S., Vetterling, W.: Numerical Recipes in FORTRAN 77: Volume 1 of Fortran Numerical Recipes: The Art of Scientific Computing, vol. 1. Cambridge University Press, Cambridge (1992)

17. Burgarth, D. Maruyama, K., Murphy, M., Montangero, S., Calarco, T., Nori, F., Plenio, M.B.: arXiv:0905.3373 (2009), Phys. Rev. A 81, 040303(R) (2010), http://arxiv.org/abs/0905.3373 